\title{
Protée
}

\section{Défendre l'irrecevable : comment l'ethos se substitue au logos dans le Liber amicorum Alain de Benoist}

\section{François-Emmanuël Boucher}

Volume 35, numéro 1, printemps 2007

Échos et résonances

URI : https://id.erudit.org/iderudit/015893ar

DOI : https://doi.org/10.7202/015893ar

Aller au sommaire du numéro

\section{Éditeur(s)}

Département des arts et lettres - Université du Québec à Chicoutimi

ISSN

0300-3523 (imprimé)

1708-2307 (numérique)

Découvrir la revue

Citer cet article

Boucher, F.-E. (2007). Défendre l'irrecevable : comment l'ethos se substitue au logos dans le Liber amicorum Alain de Benoist. Protée, 35(1), 93-101.

https://doi.org/10.7202/015893ar
Résumé de l'article

Publié en 2004, le Liber AmicorumAlain de Benoist regroupe les témoignages de plus d'une cinquantaine de penseurs qui, indépendamment de leur allégeance politique, esquissent le portrait d'un homme aux idées controversées, complexes et protéiformes dont l'évolution au cours des quarante dernières années demeure indissociable de celle de la " Nouvelle droite » européenne. L'argumentation oscille entre une défense contre des accusations " fautives " venant d'intellectuels malveillants que l'on décrit comme inféodés à l'ordre établi et l'éloge d'une pensée " libre " et " ouverte " sur une organisation politique différente de la démocratie actuelle définie comme galvanisée par la consommation. Le but sera ici d'examiner l'imbrication constante du genre délibératif dans le portrait que l'on fait d'Alain de Benoist, lequel relève traditionnellement du genre démonstratif. Que ce soit par le biais d'une anecdote sur sa jeunesse, son travail prolifique ou son " refus des modes intellectuelles ", il faudra examiner la fine articulation entre ses moeurs et sa pensée, le lien entre l'honnêteté d'un caractère et la véracité d'une doctrine, ou du moins expliquer en quoi, sur le plan rhétorique, les deux éléments semblent indissociables lorsqu'il s'agit de persuader de la valeur d'une argumentation. Finalement, il faudra expliciter en quoi l'enlacement entre le délibératif et le démonstratif donne à la vie privée une dimension civique dont les jeux de miroirs, selon les cas, discréditent ou glorifient un même individu.
Ce document est protégé par la loi sur le droit d'auteur. L'utilisation des services d'Érudit (y compris la reproduction) est assujettie à sa politique d'utilisation que vous pouvez consulter en ligne.

https://apropos.erudit.org/fr/usagers/politique-dutilisation/ 


\title{
DÉFENDRE L'IRRECEVABLE: COMMENT L'ETHOS SE SUBSTITUE AU LOGOS DANS LE LIBER AMICORUM ALAIN DE BENOIST
}

\author{
FRANÇOIS-EMMANUËL BOUCHER
}

\section{INTRODUCTION ${ }^{1}$}

J'analyse dans ce texte la manière dont on défend les positions philosophiques et surtout politiques d'Alain de Benoist dans un livre publié pour souligner son soixantième anniversaire de naissance, le 11 décembre 2003. Souvent regardé comme le doctrinaire le plus prolifique de la « Nouvelle droite ", Alain de Benoist n'a cessé d'être mêlé à d'interminables querelles depuis ses écrits de jeunesse sur la défense de l'Algérie française jusqu'à ses dernières analyses sur la seconde guerre en Iraq. Tiré à six cents exemplaires, le Liber amicorum Alain de Benoist (2004; dorénavant Liber) rassemble les textes de près de soixante de ses amis et connaissances qui tentent de montrer une autre image de ce penseur identifié à tout instant comme "l'intellectuel le plus persécuté en France» (M. Thibault, Liber: 247). De façon générale, j'essaierai de répondre à deux questions : premièrement, pourquoi la presque totalité des auteurs de ce livre concentrent leur argumentation sur la personne d'Alain de Benoist? Deuxièmement, quels sont les mécanismes argumentatifs qui sous-tendent la création de l'ethos d'une personne dont les discours sont irrecevables dans la société contemporaine?

Le problème central autour duquel s'élabore I'œuvre d'Alain de Benoist, œuvre qui s'étend sur plus d'une trentaine d'années, concerne le postulat de l'irrévocable obsolescence de l'alternative gauche/droite en tant que modèle $d^{\prime}$ intellection des différentes pensées qui englobent la sphère politique aujourd'hui. L'axiome de départ de ce penseur est le suivant. Les pensées de gauche comme les pensées de droite sont certes désormais discréditées en raison des mul- tiples faillites des systèmes idéologiques qu'elles ont justifiées au cours du xxe siècle. Mais surtout, la référence à ces modes de pensée est devenue inutile parce que le monde actuel offre une complexité sans équivalence avec le dernier siècle, complexité devant laquelle le cadre analytique qu'offre chacune des branches de cette alternative serait inopérant compte tenu de son incapacité d'en rendre compte avec exactitude.

Reste à savoir si cette volonté affichée de vouloir inscrire I'analyse à l'extérieur du territoire circonscrit par I'alternative gauche/droite ne trouverait pas déjà, elle-même, son origine chez certains doctrinaires du début du xxe siècle, doctrinaires aujourd'hui discrédités parce que, délibérément ou non, ils auraient justifié non seulement certaines pratiques condamnables, mais aussi les pires entreprises criminelles des nazis. Pour ses détracteurs, et ils sont nombreux, il n'y a pas de doute qu'Alain de Benoist ne rompt en rien avec le passé, mais réadapte pernicieusement plusieurs doctrines de droite, sans jamais extirper complètement de ses analyses une certaine conception du monde incompatible avec la bonne gouvernance et hautement hostile à la liberté.

J'analyserai donc les principales stratégies argumentatives utilisées en vue de défendre Alain de Benoist ${ }^{2}$. J'expliquerai la nature des accusations usuelles qu'on adresse aux discours «néo-droitiers » pour ensuite tacher de comprendre pourquoi les textes du Liber négligent les idées d'Alain de Benoist au profit de la construction d'un ethos de manière à souligner, en vue bien sûr de les dénoncer, plusieurs «travers» de la pensée des «intellectuels modernes », pensée perçue comme inféodée à «la triade consumérisme / règne de la 
marchandise / développement technologique» (M. Tarchi, Liber : 238). La référence systématique à l'ethos, là où prédomine l'éloquence judiciaire, c'est-à-dire un type d'argumentation qui vise à défendre un personnage, serait liée certes à I'antinomie des positions argumentatives que provoquent des débats polarisés, mais, aussi, à un refus de la discussion ou à une discussion impossible. Les auteurs du Liber, paradoxalement, dans le cas d'un livre qui vise à réhabiliter un écrivain, cherchent non pas tant à défendre la pensée d'Alain de Benoist, en multipliant les exemples prouvant que la justesse de cette dernière serait «quasi incontestable», qu'à faire apparaître par le biais d'anecdotes, d'expériences et de souvenirs les traits d'un être «complexe» à travers lesquels le lecteur ne pourra que reconnaître le portrait d'un homme "probe» et "généreux». Loin de défendre les écrits d'Alain de Benoist qui, aux dires de ses détracteurs, seraient suspects et qui, par conséquent - selon une logique de la réhabilitation -, gagneraient à être défendus, on déplace le débat en insistant sur l'ethos du personnage de sorte que le Liber vise non pas tant à justifier une position intellectuelle qu'à réaffirmer des "distinctions». Aux yeux de ses admirateurs, l'insistance sur l'ethos servirait moins à contrecarrer une argumentation défaillante et, dès lors, une réhabilitation impossible qu'à réinsérer, parallèlement ou par ricochet, un argumentaire à l'intérieur de la sphère des discours légitimes.

\section{MISE AU POINT SUR L'ETHOS \\ ET L'ETHOS PRÉDISCURSIF}

Dans un article publié en 1999, «Ethos, scénographie, incorporation », Dominique Maingueneau insiste sur l'idée que certaines définitions de base, léguées par la rhétorique antique, doivent être complexifiées si l'on tient à rendre compte $d^{\prime}$ une multitude de phénomènes modernes les plus disparates. Autour de la définition du terme d'ethos, Maingueneau propose une nouvelle catégorie - celle d'ethos prédiscursif - qui pourrait servir à l'analyse de différents problèmes, que ce soit celui entourant l'idée générale d'une réputation ou, encore, celui des conséquences, sur le plan rhétorique, de la création de personnages sociaux dont l'ethos préexisterait au discours par le fait qu'il se manifeste déjà virtuellement en tant qu'image dans tel ou tel univers médiatique.

Si l'ethos est crucialement lié à l'acte d'énonciation, écrit Maingueneau, on ne peut cependant ignorer que le public se construit aussi des représentations de l'ethos de l'énonciateur avant même qu'il ne parle. Il semble donc nécessaire d'établir une première distinction entre ethos et ethos prédiscursif. ${ }^{3}$

Dans un monde surchargé d'informations qui touchent encore plus celui ou celle qui est le moindrement médiatisé, une grande partie de ce que l'on catégorise sous le terme d'ethos préexiste de plus en plus à l'acte d'énonciation. C'est le cas, du moins, de l'ensemble du travail argumentatif que contient le Liber qui tend, justement, à réhabiliter un personnage au pouvoir de persuasion déjà limité en raison d'un ethos prédiscursif préjudiciable.

L'entreprise du Liber s'expliquerait ainsi : avant même la lecture de n'importe quel texte d'Alain de Benoist, on connaîtrait ou, du moins, serait conscient de la réputation ambivalente de ce penseur de sorte que l'objectivité de la lecture en souffrirait. Un lecteur averti ne pourrait ignorer les débats qui, depuis celui contre le Figaro magazine en 1979, enflamment sporadiquement l'espace médiatique français au sujet de la «Nouvelle droite». Indépendamment de ses positions politiques, ce même lecteur serait déjà habité par un nombre de caractéristiques sans doute stéréotypées qui accompagneraient la figure d'Alain de Benoist. En cherchant alors à s'attaquer à l'ethos prédiscursif du personnage de manière à le transformer ou, du moins, afin d'en proposer un autre plus apte à inspirer la confiance, les auteurs du Liber avancent que I'incompréhension manifestée autour $\mathrm{d}^{\prime}$ Alain de Benoist est indissociable de la méconnaissance de son caractère «exceptionnel » qui constituerait, en définitive, la plus importante des preuves en sa faveur. Là où ses idées seraient incomprises, là où l'évolution serait cahoteuse, là où mêmes plusieurs fréquentations seraient suspectes et certaines théories erronées, I'homme néanmoins resterait admirable, compte tenu de la richesse unique de son être; ethos dont la profondeur réhabiliterait ses discours qui, finalement, ne seraient qu'une extrapolation de cette complexité dans le domaine de l'énonciation. C'est pourquoi, ici, la personnalité n'ennoblit pas seulement le logos; il devient son rédempteur, son créancier rhétorique à l'intérieur de l'espace médiatique et intellectuel.

\section{LE DÉBAT D'IDÉES}

\section{ET LES DANGERS DE LA DIABOLISATION}

L'une des caractéristiques du Liber demeure la présence, chez de nombreux auteurs, de réels désaccords avec la pensée d'Alain de Benoist. En fait, rares sont ceux 
qui n'affirment pas d'emblée leur plus nette distance face à certains discours de ce penseur. On calcule par dizaines des critiques de toutes sortes, de fortes divergences, parfois même des reproches sur l'énonciation de certaines thèses si ce n'est le signe d'une totale incompréhension devant plusieurs de ses écrits:

Peu de nous, à Telos, explique Frank Adler, sympathisons avec certaines tendances des premiers ouvrages d'Alain, qui refont occasionnellement surface sous une forme plus discrète, en particulier sa fascination pour les différences biologiques qui lui fait faire cause commune avec les théoriciens de la sociobiologie et accepter l'idée de différence d'intelligence entre les différents groupes. De même, il y par exemple, au sein du comité de patronage de Nouvelle École, des membres, anciens et nouveaux, avec lesquels nous ne pourrions coopérer.

(Liber: 9)

L'enthousiasme qu'Alain de Benoist éprouve pour plusieurs formes de polythéisme ne semble pas non plus fasciner au même point tous les auteurs qui lui rendent hommage. Bien qu'il s'agisse d'un livre où l'on témoigne de son estime, les désaccords pullulent à chaque instant dans le Liber:

Les racines païennes de l'Europe, avance Hervé Coutau-Bégarie, me paraissent relever du folklore et leur apologie de l'exercice de style pour intellectuels désœuvrés. (Liber: 66)

À l'exemple de Claude Karnoouh, plusieurs auteurs de ce livre affirment qu'ils sont « loin d'être d'accord avec tous les thèmes, toutes les analyses, avec toutes les interprétations proposées dans les revues ou dans les ouvrages d'Alain de Benoist» (Liber: 136). Certes, on admire, voire ressent un engouement pour le travail accompli par le fondateur du Groupement de recherche et d'études pour la civilisation européenne (GRECE), mais cette estime partagée, qui demeure le socle du Liber, n'implique en rien une conformité du jugement ou une cohésion de I'analyse. En fait, I'un des paradoxes du livre est le suivant: quoique l'on souhaite rendre hommage au personnage, quoique l'on souhaite même transmettre cette admiration à qui voudra bien l'entendre, il demeure que cette appréciation de l'homme n'implique en rien une défense inconditionnelle de la pensée d'Alain de Benoist; plus encore, elle présuppose une prise de distance et des dissociations.

Ce groupe d'intellectuels disparates se définit ainsi par son hétérogénéité manifeste, par ses clivages et ses distan- ciations affichées. II demeure cependant qu'à l'inverse de la réaction de plusieurs de ses détracteurs, les désaccords ressentis produisent le désir non pas d'instaurer une ségrégation autour d'Alain de Benoist, mais plutôt de poursuivre le dialogue même s'il consiste à afficher des divergences. Le Liber opère donc certains clivages au sujet des critères de jugement en postulant que l'estime pour un travail intellectuel ne présuppose pas le partage des valeurs véhiculées par ce travail. En effet, par-delà ladite production intellectuelle, par-delà même la défense au nom de la liberté d'opinion, existeraient des traits de personnalité susceptibles par eux-mêmes, indépendamment de tout autre critère, de justifier le respect.

Dès lors, la différence qui explique la nature opposée des réactions serait la suivante: au contraire de ses ennemis qui «ne voient en lui qu'un propagandiste et qu'un doctrinaire qui a fait des idées un usage instrumental, caché, au service d'un projet essentiellement politique ${ }^{4}$, les auteurs du Liber posent en principe qu'Alain de Benoist est un penseur atypique le plus souvent bizarre et que c'est justement en raison de cette personnalité «complexe» qu'il est souvent incompris ou caricaturé comme un monstre. De manière beaucoup plus générale, on part de l'idée qu'il existe des différences axiologiques irréductibles entre les écrits d'Alain de Benoist et les discours d'extrême droite que l'on retrouve par exemple dans les colonnes de Minute, de Présent, de Rivarol et de National hebdo, en somme des discours que I'on retrouve chez la droite la plus vulgaire. Ainsi, sur le plan intellectuel, cette disparité cognitive accorderait une légitimité aux projets du GRECE, du moins ne rendrait pas I'entreprise ontologiquement condamnable. Entre les textes d'Alain de Benoist et $n$ 'importe quelle doctrine d'obédience populiste ou ethnocentriste, il existerait non pas une simple différence de degré, mais une incompatibilité d'essence dont le fondement se trouverait, justement, dans le caractère " aristocratique» de sa personne. À l'amalgame qu'on présuppose être le propre de la stratégie argumentative des opposants de la « Nouvelle droite», les auteurs du Liber répondent par la dissociation, par la réhabilitation négative et, finalement, par la critique de la diabolisation d'un adversaire ${ }^{5}$.

Par opposition à la figure d'un néo-nazi camouflé sous une suspecte érudition ou d'un supporteur de Le Pen, de Heider et de Bossi, d'autant plus dangereux que producteur d'une doctrine alambiquée, les auteurs du Liber présentent la figure d'un intellectuel innovateur qui refuse l'antique 
partition binaire du monde et qui s'est départi d'un bagage notionnel à jamais obsolète. Tant bien que mal, parfois en commettant des erreurs, mais toujours en évoluant, Alain de Benoist analyserait les transformations que connaît l'Europe depuis au moins 1945. Lui-même, note-t-on dans le Liber, s'est à maintes reprises métamorphosé; il a souvent changé de référence, il a même carrément critiqué certains de ses points de vue, mais jamais il n'a argumenté en tenant compte des stéréotypes que colportent, selon le Liber, les médias et les modes françaises. En fait, et c'est là que réside tout le nœud du problème autour de la figure d'Alain de Benoist, les qualités qu'admirent ceux qui lui rendent hommage s'avèrent souvent les mêmes qui alimentent la controverse et entretiennent sa mauvaise réputation. Le cas le plus typique de cette perception ambivalente reste ce désir revendiqué par Alain de Benoist de se soustraire à l'alternative gauche/droite et d'occuper des positions hétérodoxes à première vue incohérentes. Loin d'être une prouesse admirable, cette posture intellectuelle représente, pour Roger Griffin, le propre, pour ne pas dire l'essence même, d'une "fascist philosophy of history» (2000: 51). C'est le refus d'occuper une position claire et de puiser à une seule tradition qui rend Alain de Benoist beaucoup trop suspect:

The synthesis, explique Griffin dans un long article sur la « Nouvelle droite », of elements taken from the Left and the Right of the spectrum, has historically always been a feature of fascism. 6

Bien qu'elles puissent paraître comme une forme d'ouverture d'esprit, les tendances avouées d'un homme de " droite » à considérer et même à admirer certaines théories révolutionnaires de "gauche» ou, pire encore, à faire l'éloge du multiculturalisme pour contrer les contrecoups de la globalisation demeurent, pour Tamir Bar-On, un autre critique de la «Nouvelle droite», le prélude à la mise en place de politiques totalitaires. Mises à part certaines différences cosmétiques, la pensée d'Alain de Benoist reste, pour ses détracteurs, indéfendable, car elle colporte la même conception du monde qui a donné autrefois naissance au fascisme:

At the same time, despite the ND's opening to the left and greater cultural pluralism, écrit Tamir Bar-On, we can still see how a number of its authors and themes show a thread of ideological continuity with the ideas of the Conservative Revolution and "non-conformist » milieu, which clearly appealed to the Fascist and the Nazi regimes of the past. ${ }^{7}$
Dans ce contexte, le portrait que l'on trace d'Alain de Benoist, en plus d'éviter de répondre aux controverses, remplit deux fonctions précises : il réhabilite et condamne à la fois; il cherche à rétablir la réputation en postulant la grandeur aristocratique de l'homme, ce qui, du même coup, montrerait le ridicule de tous ceux qui le diabolisent en le présentant comme la continuité logique des entreprises nazies. L'ethos prédiscursif qu'offrent de lui les médias et certaines revues savantes deviendrait d'autant plus insensé que les traits derrière lesquels apparaît la figure d'Alain de Benoist le font plus humain, plus «sympathique», plus «probe», plus digne, surtout, de représenter un être « authentiquement original » selon, bien sûr, des caractéristiques que les auteurs de ce livre s'appliquent à définir en partant de ce que peut être la conduite d'un homme qui «recherche la vérité».

\section{LE SAVOIR ET L'OPACITÉ DU MONDE}

Par-delà les disputes au sujet des écrits d'Alain de Benoist, il est possible d'identifier deux modes opposés d'intellection du réel: le premier est expérimental, chaotique, disharmonieux, il fonctionne autant par bonds que par saccades, autant par zigzags que par tâtonnements, par le simple fait que la "vérité » s'apparente alors à une quête et non à une donnée concrète. Le second mode est plutôt autoritaire, déclamatoire, emphatique; il s'appuie sans cesse sur des révélations ou sur «le sacre du présent», présupposant que la vérité préexiste à l'acte d'intellection. Tandis que l'un regarde la situation présente de l'homme comme un fait énigmatique et, par conséquent, invite à l'exploration de la complexité du monde, l'autre postule qu'à l'extérieur d'un système de valeurs très précis se déploie le territoire de l'hérésie et de l'indéfendable.

Le problème jamais soulevé par le Liber est de savoir si la fameuse hétérodoxie d'Alain de Benoist en est vraiment une; si, par exemple, faire l'éloge de la colonisation française est en soi une forme d'exploration de la complexité du monde; si promouvoir les dernières théories sur les inégalités intellectuelles à partir de critères génétiques est faire preuve d'une plus grande ouverture sur l'énigmaticité de la condition humaine; si propager les pires formes d'anti-américanisme est en soi un acte philosophique qui présuppose une saisie inédite du réel; ou, encore, si l'expérimentation cognitive justifie la promotion de la suppression des droits de I'homme au nom d'utopies écologistes. II va sans dire que la défense de la liberté humaine implique la lutte con- 
tre la barbarie qui triomphe trop souvent un peu partout. Cependant, il reste que le meilleur antidote à ce problème ne se trouve peut-être pas dans les écrits de la «Nouvelle droite ${ }^{8}$.

C'est néanmoins à partir de cette donnée que doit se comprendre la première caractéristique de la figure d'Alain de Benoist: celle d'un «éveilleur», dont les écrits ont provoqué chez les lecteurs qui lui rendent hommage des ouvertures insoupçonnées vers de nouveaux horizons.

Avec certains autres, raconte Fabrice Valclérieux, tu fus un «éveilleur» et je t'en suis au plus haut point reconnaissant.

(Liber: 250)

Aujourd'hui, confie Júlio Prata Sequeira, je peux affirmer que ce livre [Vu de droite] a changé ma vie. II m'a apporté une nouvelle vision du monde, de nouveaux auteurs et surtout la connaissance d'Alain de Benoist. (Liber: 205)

Pour moi, avoue à son tour Philippe Lestienne, tu as été l'Éveilleur, et puisque l'occasion m'en est donnée, je veux t'en remercier. (Liber: 153)

Prisonnier d'une Europe qui se cherche et d'une France, surtout, qui «s'écroule» sous le poids d'une mémoire dont la réminiscence tient lieu de pensée, Alain de Benoist, souligne Liber, fait figure d'exception tant «son énergie, sa très vive intelligence, ses vastes connaissances » (T. Molnar, $L i$ ber: 188) contrastent avec la «stérilité» des milieux intellectuels traditionnels. On le présente à maints endroits comme « un grand universitaire» (O. Dard, Liber: 72), « un vrai maître» (M. Christadler, Liber: 63), " un champion de ce que devrait être un homme qui a choisi de se consacrer entièrement aux idées» (A. Campi, Liber: 44). Son «magnétisme » est tel que même des auteurs qui, en raison d'a priori politiques différents, devraient s'avérer ses adversaires, lui témoignent une reconnaissance pour "sa parfaite probité», pour "sa très grande hauteur de vue»(C. Gambescia, $L i-$ ber: 82). En fait, l'aura qui entoure le personnage resplendit encore plus lorsqu'on le rencontre: la présence réelle de sa personne efface tous les désaccords que l'on pourrait avoir ou, du moins, les rend secondaires. Encore une fois, c'est l'éloge du personnage qui prend le dessus sur le débat d'idées; c'est la puissance de l'ethos qui parvient à pallier les défaillances du logos.

J'ai abordé cet homme, écrit Frank Adler, en intellectuel de gauche qui a appris à connaître, à admirer et à respecter Alain de Benoist. (Liber: 10)
Jusqu'au printemps 1986, poursuit Claude Karnoouh, j'avais cru qu'Alain de Benoist était un vilain personnage, un chantre d'une extrême droite xénophobe, colonialiste, patriotarde, vichyssoise et revancharde. (Liber: 130)

Mais, après sa première rencontre avec le fondateur du GRECE, il a été terrassé par l'inexactitude de ses jugements: [...] à partir de cette rencontre, continue Karnoouh, je commençai à lire les revues que dirigeait Alain de Benoist. Qu'aije découvert? En premier lieu, que bien des accusations dont elles étaient l'objet étaient erronées, voire totalement fausses.

(Liber: 135)

L'écart entre le personnage et la réputation que colportent les médias français devient, dans le Liber, un phénomène inquiétant, porteur de significations sur le monde actuel et dont plusieurs s'efforcent de thématiser les causes. En soulignant sous tous les angles la "fausseté» des discours qui construisent l'ethos prédiscursif d'un individu dans un univers médiatique donné, le Liber cherche à éveiller la suspicion vis-à-vis du pouvoir sans précédent des médias, du témoignage des experts, des médiatisations des personnalités, si ce n'est de la possibilité de transmettre un discours savant dans un espace public. La défense d'Alain de Benoist s'apparente dès lors à une mise en procès des mécanismes qui, depuis deux siècles, ont permis la montée de la bourgeoisie et I'apparition de I'intellectuel ${ }^{9}$. La valorisation de la personnalité complexe d'Alain de Benoist - son ethos chatoyant, alambiqué et susceptible de connaître d'étranges métamorphoses - devient la critique de la simplification que plusieurs auteurs du Liber posent maintenant à la base de la médiatisation de la pensée dans le monde globalisé, couvert virtuellement en information continue. Étranger par essence à cet univers «vulgaire» et "mercantile», Alain de Benoist serait en quelque sorte le premier dissident $d^{\prime}$ un système inquisitorial où l'inactuel deviendrait l'ennemi numéro un.

\section{L'ETHOS DE LA DISTINCTION \\ OU L'ARISTOCRATIE DE LA PENSÉE}

$C^{\prime}$ est toujours à partir des critères d'évaluation de la complexité du monde que s'organise la défense du personnage d'Alain de Benoist. En même temps que l'on insiste sur la nature «mensongère » de sa réputation, en expliquant que le problème ne se règle pas par la diabolisation, plusieurs auteurs du Liber essaient aussi d'élargir le débat en trouvant 
une filiation historique à l'ethos d'Alain de Benoist et, encore, en montrant comment il s'oppose à tel ou tel idéaltype. Toujours prêt, écrivent les auteurs du Liber, à se remettre en question, «à mépriser le confort des certitudes définitives [...] avec la volonté d'explorer plus avant le champ infini de la connaissance» (J.-M. Zagamé, Liber: 259), ce penseur «atypique» a "évolué contre la mode» 10 , traduisant ainsi à tous moments "sa volonté de rester vivant, alerte, curieux et ouvert sur le monde»(A. Campi, Liber: 45), si ce n'est son refus « du dogmatisme et de l'accaparement de l'esprit par l'idéologie » (ibid.). Là où, suggère-t-on, l'ethos prédiscursif médiatisé plaque sur la figure d'Alain de Benoist le personnage du doctrinaire intransigeant et cruel, certains auteurs du Liber s'efforcent paradoxalement de démontrer, en insistant sur ses perpétuelles métamorphoses, I'absence, autant chez lui que dans ses écrits, d'un quelconque système d'idées cohérent que l'on pourrait objectiver sans raccourcis malhonnêtes. En effet, I'un des arguments utilisés pour le défendre consiste à montrer qu'Alain de Benoist se définit d'abord par une liberté d'esprit qui, par principe, est incompatible avec la création d'idéologies. L'ethos possède une telle profondeur, une telle variété de coloris inusités que vouloir le réduire à une personnalité de fanatique soulignerait un manque de jugement indigne $d^{\prime} u n$ grand herméneute. «Il y a belle lurette, assure Gérard Landry, que le lecteur honnête des écrits d'Alain de Benoist ne peut plus coller une étiquette sur l'œuvre et sur l'homme» ( $\mathrm{Li}$ ber: 148). Explorateur de savoirs disparates, hostile «au compartimentage rigide des disciplines »(A. Campi, Liber: 45), Alain de Benoist, assure Jean-Marcel Zagamé, serait l'un des seuls de son époque qui «a rejoint la tradition des grands humanistes de la Grèce et de la Renaissance italienne» ( $\mathrm{Li}$ ber: 260). Sans jamais expliquer en détail ses positions, on avance maintenant que ce penseur n'aurait d'équivalent que Michel-Ange ou Giordano Bruno. À leur exemple, et contrairement au "jargonneur universitaire moderne» (P. Lestienne, Liber: 152), prêt à maints compromis afin de $\mathrm{s}^{\prime}$ assurer « une réussite mondaine ou mercantile» ( $\mathrm{H}$. CoutauBégarie, Liber: 66), Alain de Benoist a toute sa vie refusé "de grimper dans l'échelle des valeurs bourgeoises» (J. Marlaud, Liber: 161). Sa personnalité devient d'autant plus remarquable qu'elle reste incompatible avec la société française contemporaine. En fait, pour quiconque connaît le moindrement le contenu des publications d'Éléments ou de Nouvelle école, la peinture que I'on présente d'Alain de
Benoist devient rapidement I'antithèse parfaite du personnage que I'on thématise depuis au moins vingt ans sous l'appellation de «bourgeois». Cet idéal-type rendrait compte en quelque sorte de la «vulgarité » de la société actuelle en raison de sa haine déclarée du gratuit et de son égoïsme calculateur. Parallèlement à la défense d'Alain de Benoist, le Liber poursuit la polémique avec ceux-là même que ce livre s'efforce de contredire en érigeant en idéal ou, du moins, en une personne digne d'admiration, l'opposé exact de ce que I'on considère comme l'intellectuel moderne admiré aujourd'hui par les universitaires et les médias hexagonaux. À l'ombre de la peinture d'Alain de Benoist, on en vient à dessiner négativement l'ethos de celui qui ne s'offusquerait pas d'un système où la «simplification» et les plus «bas intérêts » assureraient une réussite parfaite, un prestige et même, parfois, la reconnaissance populaire.

Les trop rares fois où j'ai eu l'occasion d'échanger des idées avec lui, raconte Kostas Mavrakis, j'ai senti ce qu'est l'ethos propre au philosophe, à savoir le souci de la vérité sans concession et la volonté d'aller jusqu'au bout des choses. Sa vie publique confirme que le courage et l'honnêteté vont de pair avec cette attitude. (Liber: 178)

Le Liber non seulement ne se lasse de souligner ses similitudes avec les « humanistes renaissants», mais s'applique aussi à décrire minutieusement son arbre généalogique, à rappeler l'assassinat d'une partie de ses ancêtres à l'époque de la Terreur et à expliquer les caractéristiques de sa grand-mère, "petite-nièce du peintre Gustave Moreau » qui fut «la secrétaire du célèbre Gustave LeBon»(L. Pauwels, Liber: 198). Descendant ainsi d'une famille dont le premier ancêtre identifié - Gobert van Steelant - «vivait en 790 en Hollande» (ibid.: 197), Alain de Benoist, confie Jean Jouven, possède toujours "une dignité sénatoriale et juvénile à la fois » ( $\mathrm{Li}$ ber: 117). « $C^{\prime}$ est un très grand penseur, gracieux, actif, d'une distraction concentrée, une aristocratie qui s'efface généreusement» (M. Christadler, Liber: 55). Amoureux des livres, «travailleur infatigable» doté $d^{\prime}$ une «immense érudition », soucieux finalement davantage de la "vérité » que de la réussite mondaine, Alain de Benoist est défini par ce goût du sacrifice, par le don de soi, par sa quête désintéressée. À I'exemple de ce que racontent les grandes traditions au sujet des philosophes d'autrefois, à l'exemple des valeurs que lui ont léguées ses ancêtres aristocratiques, la richesse matérielle n'a jamais été au centre de sa vie. 
C'est, je crois, résume Jean Laloux, cette générosité-là qui explique ta détestation de la figure du bourgeois et de la société qui l'a engendrée; tu en es tout simplement l'antithèse.

(Liber: 146)

Ainsi, l'ethos d'Alain de Benoist se construit sur différentes oppositions dont les figures qui servent de repoussoir ont des caractéristiques déjà explicitées et critiquées dans les écrits de la «Nouvelle droite». Du «bourgeois», par exemple, qui, selon le GRECE, thésaurise et ne vit que pour le gain matériel, se détache la figure stéréotypée du grand aristocrate généreux dont «le mode de vie seigneurial [serait] fait de dons, de prodigalité, de dépenses sans compter, de prédation comme générosité, de gratuité dans tous les sens du mot» ${ }^{11}$. À l'universitaire spécialisé, s'oppose l'érudit «multidisciplinaire», "l'humaniste renaissant», l'héritier du «philosophe grec» qui erre dans sa maison où se trouve "l'une des plus importantes bibliothèques privées du monde » (J. Marlaud, Liber: 160). Enfin, en face du militant hargneux des droits de l'homme, de la «meute des sycophantes» (J.M. Zagamé, Liber: 259) tétanisée par le «credo des idées reçues", des "néojacobins centralisateurs» (M. Tarchi, Liber: 240), grands supporteurs des «dictatures molles», se met en place la figure d'un être qui se questionne et pour qui I'acte de rendre compte de l'opacité du monde demeure un geste qui ne saurait être rémunéré.

\section{CONCLUSION}

La figure discursive d'Alain de Benoist qui se retrouve dans le Liber se dessine ainsi à l'intérieur d'un univers doxique qui déborde de loin cette publication. En effet, en raison de polémiques lointaines, préexiste à cette tentative de défense un nombre de concepts antithétiques, de repoussoirs notionnels, d'éléments sous-entendus déjà connotés, sémantiquement lourds d'a priori, dont on présuppose la connaissance chez le lecteur averti. Loin, cependant, d'être spécifique au Liber, et il faut le souligner, cette particularité est le propre de la construction d'un ethos en rhétorique judiciaire: I'insistance sur les caractéristiques d'une personne défendable ou non, sur les mœurs d'un penseur, critiquables ou pas, en somme l'insistance sur le caractère de n'importe quel locuteur implique l'existence d'arguments disparates dont les logiques sont irréductibles les unes aux autres, mais à partir desquelles se construit la figure de telle ou telle personne. Ainsi, l'ethos est intimement lié au processus d'argumenta- tion par le fait qu'il est en lui-même polémique, c'est-à-dire à l'opposé d'une figure objectivement neutre qui rendrait compte du caractère intime d'un être humain. L'ethos est construit par le discours, de même qu'il répond à un argumentaire qui le précède et qui lui prête sa signification. Sans être imbriqué dans le logos ou le pathos, il leur est indissociable. En fait, c'est là que se trouve le pari d'une argumentation où l'ethos prédomine largement. Il y aurait non seulement un transfert de valeur, mais aussi des interconnexions qui présupposeraient la transmutation inévitable de l'authenticité d'un personnage en une légitimité discursive.

C'est pourquoi le caractère aristocratique attribué à Alain de Benoist, son goût de la «liberté», son « hétérodoxie», en somme l'ethos que trace de lui le Liber est indissociable d'une vision précise de la société. L'anti-américanisme du GRECE, par exemple, de même que l'inquiétude que manifestent plusieurs auteurs d'Éléments, lors de chaque publication, à l'égard de toute forme d'universalisme abstrait ou d'une Europe ultra-libérale, gagneraient à être analysés à partir de la construction d'un modèle anthropologique dont l'ethos d'Alain de Benoist serait le paradigme. Ce parallèle permettrait de mieux comprendre pourquoi, au débat gauche/droite, c'est-à-dire au spectre du politique où prédominent les différentes «valeurs bourgeoises», la « Nouvelle droite» cherche par tous les moyens à y opposer une autre réflexion à partir de différents thèmes, que ce soit ceux d'autonomie, de subsidiarité, de droit des cultures ou de fédération des peuples. Le modèle anthropologique - le nouvel homme dont I'ethos d'Alain de Benoist propose un aperçu - se situerait ainsi par-delà et surtout paradoxalement en avant, malgré de lointaines filiations, des modèles d'une «humanité satisfaite ». Par conséquent, il légitimerait par lui-même, du moins selon le point de vue du Liber, la recherche d'une autre organisation sociale où triompheraient le "don de soi», I'«altruisme», le "gratuit», en gros les caractéristiques que I'on présuppose naturelles à l'aristocratie. La «complexe humanité » que l'on prête à Alain de Benoist non seulement permet d'éviter le débat et de se soustraire à un véritable travail d'argumentation qui impliquerait le questionnement de certaines positions philosophiques mais assure aussi, indépendamment du fait qu'on se refuse à les défendre directement, une sanctification de ses innombrables théories. Pour les auteurs du Liber, le caractère exceptionnel que l'on prête à un personnage autorise finalement toutes les formes de réflexion politique, comme si I'humanité d'un ethos deve- 
nait le gage non pas tant de la véracité de la pensée d'un être en particulier, mais de l'authenticité de la démarche et de la validité de toutes les intuitions qui précèdent leur formulation.

\section{N OTES}

1. Une première version de ma réflexion a été présentée en anglais, grâce à un financement du Collège militaire royal du Canada, au colloque de I'International Society for the History of Rhetoric qui s'est tenu à I'University of Southern California (Los Angeles, Californie, 13-16 juillet 2005). 2. Rappelons qu'A. de Benoist n'est pas le seul auteur à être au centre de ce type de controverse. Plusieurs écrivains, philosophes et artistes du Xxe siècle, que l'on classe habituellement à droite, sont souvent soit considérés comme des génies ou des innovateurs audacieux, soit regardés comme des fascistes ou des êtres tordus dont la fréquentation demeure peu recommandable. Voir, à ce sujet, l'article de Caldwell (2005) sur les débats autour de C. Schmitt ainsi que de sa contribution aux théories politiques de son époque. Voir aussi P. Muray (2001-2002; 2002).

3. D. Maingueneau, «Ethos, scénographie, incorporation », dans Amossy (dir.), 1999: 77-78.

4. A. Campi (Liber: 43). Au sujet de la défense d'A. de Benoist, voir aussi Taguieff: "Singulier objet de controverse: la N.D. [nouvelle droite] est assimilée à l'extrême droite par nombre de journalistes, stigmatisée en tant que néo-nazie par certains militants anti-fascistes, rejetée par la droite libérale pour son anti-américanisme radical, dénoncée comme procommuniste ou crypto-gauchiste par les dirigeants lepénistes ou certains idéologues traditionalistes catholiques, accusée de fournir des armes idéologiques à la droite conservatrice, soupçonnée de faire partie d'une internationale "nationale-bolchevique", suspectée enfin de vouloir séduire l'intelligentsia de gauche en lui offrant largement les colonnes de ses revues. La confusion est manifeste» (1994: iii). Voir enfin Piccone, I'un des admirateurs d'Alain de Benoist aux États-Unis (1993-1994: 6).

5. L'amalgame et la dissociation sont au cœur des stratégies argumentatives qui entourent le débat sur l'extrême droite et la "Nouvelle droite» en France. Voir P. Breton, "La préférence manipulatoire», dans Bonnafous et Fiala (1999: 106, 112 et suiv.).

6. «La synthèse d'éléments pris à la fois à la gauche et à la droite du spectre politique a historiquement toujours été une caractéristique du discours fasciste». (Griffin, 2000: 48; ma traduction).

7. «Au même moment, malgré que la Nouvelle Droite valorise certaines doctrines de gauche ainsi que le multiculturalisme, nous pouvons quand même remarquer que nombre d'auteurs étudiés et de sujets abordés indiquent une filiation idéologique avec les idées propres à la Révolution conservatrice et propres au milieu non conformiste de l'entre-deux guerres, thèmes qui ont fasciné les régimes fascistes et nazis du passé ». (Bar-On, 2001 : 346; ma traduction)

8. En ordre, je renvoie à F. Laroche ( pseud. d'Alain de Benoist) (1963); F. Laroche et F. d'Orcival (1965); A. de Benoist $(1998 ; 2003 ; 2004)$.

9. Sur les origines et la montée de l'intellectuel dans l'espace public, voir surtout Habermas (1986) et Charle $(1990 ; 2001)$.

10. C. Karnoouh (Liber: 137); voir aussi Amossy qui explique que cet argument est sans cesse utilisé pour défendre les positions de la « Nouvelle droite», dans son article "Sociocritique et argumentation »: "Rompant avec son passé d'activiste, Benoist se désolidarise de tout parti et passe à une démarche dite "métapolitique". Contrairement à ses détracteurs qui ne voient dans cette conversion qu' un engagement de tactique, Alain de Benoist prend le plus grand soin de justifier la séparation des champs politique et intellectuel. Cette division, pour lui salutaire, explique à la fois son positionnement propre et la nature de son discours» (1992: 33).

11. Définition de l'aristocratie selon A. de Benoist (1992); voir aussi J. Marlaud (Liber: 167). 


\section{RÉFÉREN CES BIBLIO g RAPHIQ U ES}

AMOSSY, R. [1992] : "Sociocritique et argumentation: I'exemple du discours sur le "déracinement culturel" dans la Nouvelle droite», La Politique du texte, Lille, Presses universitaires de Lille, 29-50;

[2000] : L'Argumentation dans le discours. Discours politique, littérature d'idées, fiction, Paris, Nathan.

AMOSsY, R. (dir.) [1999] : Images de soi dans le discours. La construction de l'ethos, Lausanne, Delachaux et Niestlé.

ARISTOTE [1991] : Rhétorique, trad. de C.-É. Ruelle et P. Vanhemelryck, Paris, Le livre de poche.

BAR-ON, T. [2001]: "The Ambiguities of the Nouvelle droite, 19681999", The European Legacy, vol. 6, no 3, 333-351.

BENOIST, A. de [1989] : «Contre Hayek » (tiré d'une conférence qui a eu lieu à Nice). En ligne: http://www.alaindebenoist.com/pages/textes.php? cat= livres\&lang=fr (page consultée le 15 février 2007);

[1992] : «Le Bourgeois», Éléments, nº 72, janvier, 11-16. En

ligne: http://www.alaindebenoist.com/pages/textes.php?cat=orientation \&lang=fr (page consultée le 15 février 2007);

[1994] : «Communautariens vs libéraux », Krisis, nº 16, juin, 229. En ligne: http://www.alaindebenoist.com/pages/textes.php?cat= orientation\&lang=fr (page consultée le 15 février 2007);

[1998]: The Study of Intelligence and the IQ Controversy. A Bibliographical Introduction, 1869-1997, Washington, Institute for the Study of Man;

22 ;

[1999] : «Un mot en quatre lettres», Éléments, no 95, juin, 18-

[2002]: «La querelle des nouveaux réactionnaires. Entretien avec Alain de Benoist ». En ligne : http://www.alaindebenoist.com/pages/ textes.php?cat=entretiens\&lang=fr (page consultée le 15 février 2007);

- [2003] : « Les coulisses de la guerre d'UBush », Éléments, nº 108, janvier, 4-9;

[2004]: Au-delà des droits de l'homme. Pour défendre les libertés, Paris, Krisis.

Bonnafous, S. et P. Fiala (dir.) [1999]: "Argumentation d'extrêmedroite», Mots. Les langages du politique, no 58.
CALDWELL, P. C. [2005] : "Controversies over Carl Schmitt; A Review of Recent Literature", The Journal of Modern History, vol. 77, no 2, 357-387. CAPLOW, T. [1997] : "Beyond Coca-Cola : Europe and the American way", La Revue Tocqueville, vol. XVIII, no 2, 157-161;

[2001] : «Le Léviathan passé au crible: une évaluation de l'État américain au seuil du XXI siècle», La Revue Tocqueville, vol. XXII, nº 1, 13-44.

CHARLE, C. [1990] : Naissance des intellectuels 1880-1900, Paris, Minuit; [(1996) 2001] : Les Intellectuels en Europe au XIXe siècle: essai d'histoire comparée, Paris, Seuil.

DECLERCQ, G. [1992] : L'artd'argumenter. Structures rhétoriques et littéraires, Bruxelles, Éd. de l'Université de Bruxelles.

Éléments [2001] : Le IIle Millénaire a commencé le 11 septembre, no 103, décembre.

GRIFFIN, R. [2000]: "Between metapolitics and apoliteia: the Nouvelle droite's strategy for conserving the fascist vision in the interregnum", Modern \& Contemporary France, vol. 8, no 1, 35-53.

Habermas, J. [1986] : L'Espace public, Paris, Payot.

LAïDI, Z. [2000] : Le Sacre du présent, Paris, Flammarion.

LAROCHE, F. (pseud. d'A. de Benoist) [1963] : Salan devant l'opinion, Paris, Éd. Saint-Just.

LAROCHE, F. et F. d'Orcival [1965] : Le courage est leur patrie, Paris, Éd. Saint-Just.

MARMIN, M. (dir.) [2004] : Liber amicorum Alain de Benoist, Paris, Les amis d'Alain de Benoist.

MURAY, P. [(1991) 2002] : L'Empire du Bien, Paris, Belles Lettres; - [2001-2002] : Après l'histoire, 2 tomes, Paris, Belles Lettres.

Perelman, C. et L. OlbreChTs-TyteCA [1992] : Traité de l'argumentation, Bruxelles, Éd. de l'Université de Bruxelles.

PICCONE, P. [1993-1994] : "Confronting the French new-right: old prejudices or a new politica paradigm", Telos, hiver et printemps, no 98-99, 3-20.

TAgUiefF, P.-A. [1994]: Sur la Nouvelle droite. Jalons d'une analyse critique, Paris, Descartes \& Cie. 